\title{
Iodocyclization of Diallyl-Dihydroxy Naphthalenes Using $N$-Iodosuccinimide vs Molecular Iodine in Aqueous Micelle
}

\author{
Pradipta Kumar Basu*, Amrita Ghosh \\ Department of Chemistry, Hooghly Mohsin College, Chinsurah, India \\ Email: ${ }^{*}$ pkbasu74@gmail.com
}

Received February 7, 2013; revised March 20, 2013; accepted April 6, 2013

Copyright (C) 2013 Pradipta Kumar Basu, Amrita Ghosh. This is an open access article distributed under the Creative Commons Attribution License, which permits unrestricted use, distribution, and reproduction in any medium, provided the original work is properly cited.

\begin{abstract}
A number of doubly-fused [1,2] naphthodifurans have been synthesized from the corresponding diallyl-dihydroxy naphthalene precursors involving $N$-iodosuccinimide in acetonitrile and also by molecular iodine in aqueous micelle using CTAB as surfactant. The electrophilic cyclization occurs regioselectively by a 5-exo-trig mode of cyclization.
\end{abstract}

Keywords: Di-Allyl-Dihydroxy Naphthalene; $N$-Iodosuccinimide; CTAB; Micelle; 5-Exo-Trig Mode; Regioselective Cyclization

\section{Introduction}

Compounds with a naphthalene moiety are biologically active and are found to show antimicrobial activities [3, 4], anti-inflammatory effect [5], and some naphthalene derivatives are useful as antipsychotic drugs [6] and enzyme inhibitors [1]. The presence of benzofurans in the heterocyclic compounds is worth mentioning and some of them are potentially bioactive and such effects include anti cancer, anti HIV and antimicrobial activities [2,7-16].

Although the synthesis of bioactive heterocycles by metal-annulated cyclization [17-23] procedure is well known in the literature, iodonium assisted heterocyclization is also an efficient synthetic route. The iodinated organic molecules may act as useful intermediates as the iodine can be replaced by other nucleophiles. This is an attractive alternative to organometallic chemistry.

To our knowledge, there is no report of doubly fused [5,6] heterocyclic compounds from the corresponding diallyl-dihydroxy naphthalene precursors involving $N$ iodosuccinimide as the reagent and also by molecular iodine- mediated 5-exo-trig cyclization in aqueous micelle using surfactant. The efficacy of the reagent $\mathrm{N}$-iodosuccinimide and surfactant-catalyzed iodine-mediated cyclization in aqueous media prompted us to undertake a comparative study to develop a useful strategy leading to doubly fused benzofuran ring systems. Herein, we report

"Corresponding author. our results.

\section{Results and Discussion}

The required precursors $\mathbf{3 a}, \mathbf{3 c}$ and $\mathbf{3 e}$ for this purpose were prepared according to the standard published literature procedures [24,25] from the corresponding 2,3dihydroxy naphthalene (1a), 2,7-dihydroxy naphthalene (1b) and 1,5-dihydroxy naphthalene (1c) respectively by allylation followed by Claisen rearrangement. The other precursors (3b, 3d and 3f) were also accessed employing a similar protocol and characterised by spectral analyses.

Although there are reports [26-29] in the literature for the synthesis of a variety of heterocyclic compounds using $N$-iodosuccinimide, none reported the synthesis of bis fused naphthodifurans by this methodology. We, therefore, initiated our investigation with the reaction of 1,4-diallylnaphthalene-2,3-diol (3a) and $N$-iodosuccinimide in acetonitrile solvent at $0^{\circ} \mathrm{C}-5^{\circ} \mathrm{C}$.

The substrate 3a was subjected to react under standard reaction condition with $\mathrm{N}$-iodosuccinimide in acetonitrile solvent at $0^{\circ} \mathrm{C}-5^{\circ} \mathrm{C}$ for $1 \mathrm{~h}$ and then at room temperature for $3 \mathrm{~h}$. A solid was isolated in $75 \%$ yield. Its spectral data indicated that this could be tentatively assigned the naphthodifuran structure 4a. The ${ }^{1} \mathrm{H}-\mathrm{NMR}$ of $\mathbf{4 a}$ of showed two signals at $\delta 3.37$ and 3.68 as two doublet of doublets with coupling constants 15.6 and $9.6 \mathrm{~Hz}$ due to diastereotropic methylene protons of the dihydrofuran 
rings. The other four methyene protons directly attached to $\mathrm{CH}_{2} \mathrm{I}$ appeared as multiplets at $\delta 3.39-3.42(2 \mathrm{H})$ and $\delta$ $3.54-3.60(2 \mathrm{H})$. The two- $\mathrm{CH}$-protons of the dihydrofuran rings directly attached to $\mathrm{CH}_{2} \mathrm{I}$ appeared as a multiplate at $\delta 5.12-5.18$. The reaction under the same condition was extended to other substrates $\mathbf{3 ( b - f )}$ to give the products $\mathbf{4 ( b - f )}$ in $72 \%$ - $80 \%$ yields (Schemes 1-3).

From the mechanistic point of view two alternative structures are possible for the products of iodocyclization, either the structure 4 by 5-exo-trig or 6 by 6-endo-trig mode. To unambiguously establish the structure of the products we have subjected the product $\mathbf{4 a}$ to dehydroiodination condition by treating the product $\mathbf{4 a}$ in ethanolic $\mathrm{KOH}$ under reflux to afford the product $5 \mathrm{a}$. The ${ }^{1} \mathrm{H}$ NMR of the product $5 \mathrm{a}$ is different from that of the cyclized product 4a and much more simplified in 5a showing a singlet at $\delta 2.56$ for two methyl protons of newly formed two methyls by dehydroiodination followed by isomerisation of the newly generated exocyclic double bonds of the two furan rings (Scheme 1). If the product of cyclization of the substrate $3 \mathbf{a}$ were $6 \mathbf{a}$ then that after dehydroiodination would have afforded $7 \mathbf{a}$. The formation of 5a from 4a, therefore, definitely establishes the structure of the product of iodocyclization of the substrate $3 \mathbf{a}$ as naphthodifuran 4a. This was further supported by the failure of the product $\mathbf{4 b}$ to undergo dehydroiodination under the aforesaid reaction condition as there is no scope for dehydroiodination in case of $\mathbf{4 b}$. If the structure of the product of iodocyclization was $\mathbf{6} \mathbf{b}$ that should have afforded $\mathbf{7 b}$.

The dehydroiodination of the other products $\mathbf{4 c , d}$ and 4e,f also afforded the naphthodifurans 5c,d and 5e,f showing the formation of these products $4 \mathbf{c}, \mathbf{d}$ and $\mathbf{4 e , f}$ from the substrates $\mathbf{3 c}, \mathbf{d}$ and $\mathbf{3 e , f}$ exclusively by a 5-exotrig mode of cyclization in preference to 6-endo-trig mode of cyclization (Schemes 2 and 3).

There are only few reports of the iodine-mediated heterocyclization in aqueous media $[30,31]$ and also in organic media using additives [32,33] such as Envirocat EPZ-10 ${ }^{\mathrm{R}}$ or $\mathrm{SnCl}_{4}$ [34-36]. The limitation of the traditional methods may be overcome by using surfactants as cosolvents. Surfactants contain both hydrophobic groups (their lypophilic tails called micelles) and hydrophobic groups (their heads). A large volume of literature is available on the use of surfactants as cosolvents for carrying out a variety of reactions in aqueous media [37-44]. The surfactants (cationic, anionic and neutral) form micelles in protic sovents especially in water. The micelles act as a micro reactor to increase the reaction rates. Selforganized aggregates, such as aqueous micellar medium bind the insoluable organic substrates accommodating their hydrophobic tail in the mecellar interior and placing their polar head at the water micelle interface. This changes the physical properties, quantum efficiency and the reactivity of the substrates thus accelerates many organic reactions in aqueous micellar media. Recently, Majumdar and co-workers reported [45] the synthesis of angularly fused furano pyrone/coumarin and quinolone derivatives by using surfactants in water and this is the first report described in literature. We, therefore, turned our attention to investigate the electrophilic iodocyclization of the precursors 3(a-f) by molecular iodine-mediated reaction in the presence of CTAB as surfactant to evaluate application of this methodology. Our initial attempt to obtain

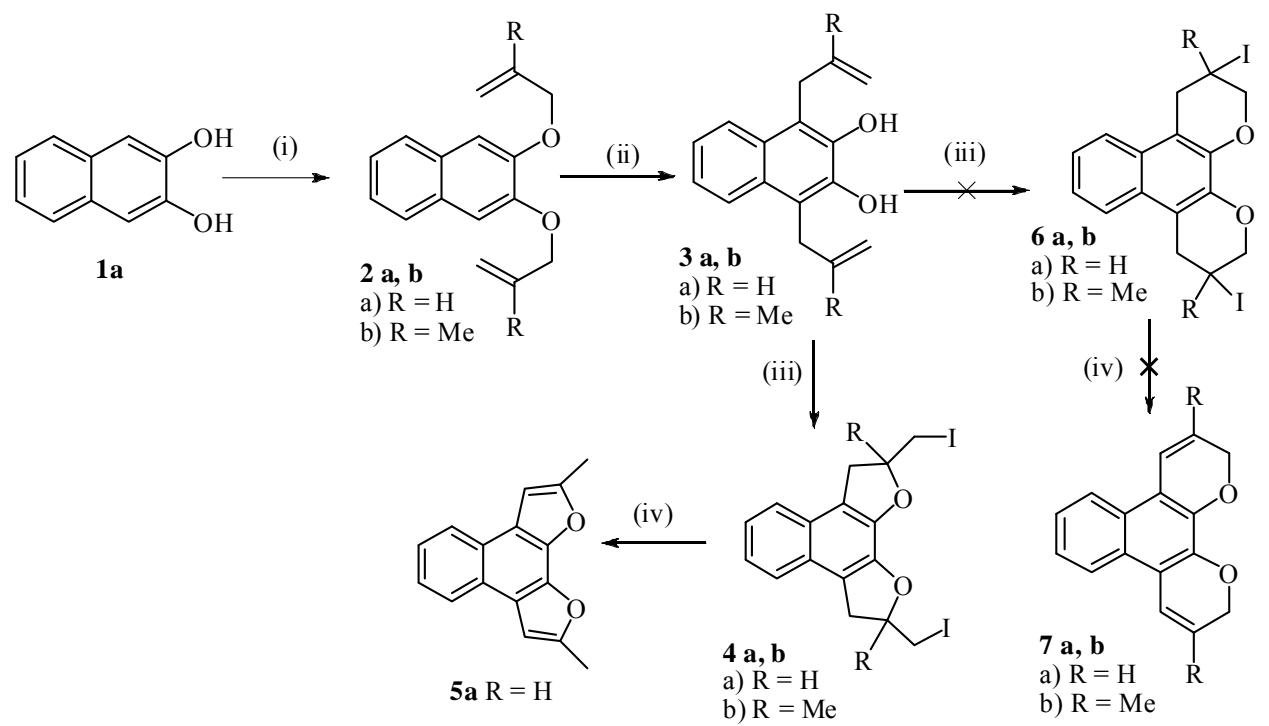

Scheme 1. Reagents and conditions: (i) Allyl bromide or 3-chloro-2-methyl-1-propene, acetone, $\mathrm{K}_{2} \mathrm{CO}_{3}$, $\mathrm{NaI}, \mathrm{reflux}, 12$ - 15 h; (ii) o-DCB, reflux, 8 - 10 h; (iii) Condition A: substrates 3a,b (1.0 mmol), NIS (2.0 mmol), MeCN, 0 ${ }^{\circ}$-r.t, 4 h; Condition B: substrates 3a,b (0.5 mmol), I (1.5 equiv.), CTAB (0.30 mmol), $\mathrm{H}_{2} \mathrm{O}$ (10 ml.), r.t, 8 h; (iv) KOH (2.0 mmol), EtOH, reflux, 2 - 3 h. 


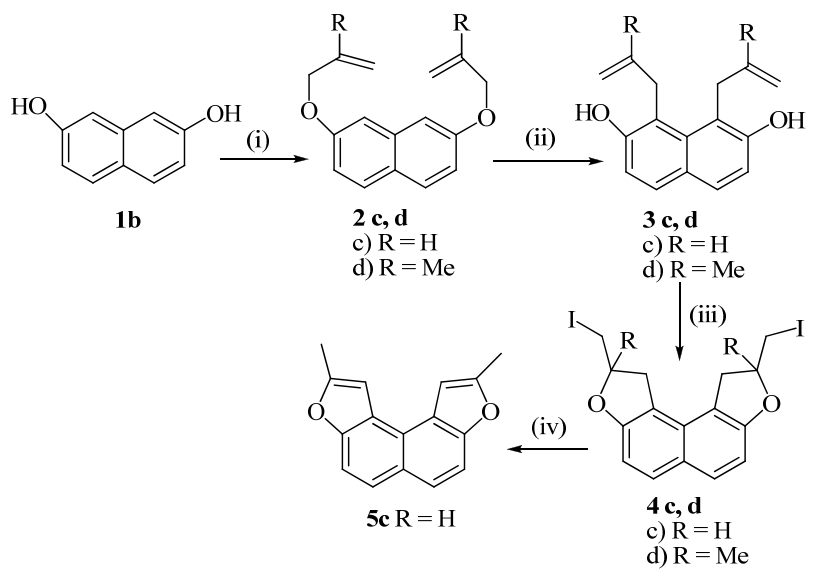

Scheme 2. Reagents and conditions: (i) Allyl bromide or 3-chloro-2-methyl-1-propene, acetone, $\mathrm{K}_{2} \mathrm{CO}_{3}$, NaI, reflux, 12 - 15 h; (ii) o-DCB, reflux, 8 - 10 h; (iii) Condition A: substrates 3c,d (1.0 mmol), NIS (2.0 mmol), MeCN, $0^{\circ} \mathrm{C}$-r.t, 4 h; Condition B: substrates 3c,d (0.5 mmol), $I_{2}$ (1.5 equiv.), CTAB (0.30 mmol), $\mathrm{H}_{2} \mathrm{O}$ (10 ml.), r.t, $8 \mathrm{~h}$; (iv) KOH (2.0 mmol), EtOH, reflux, 2 - 3 h.<smiles>Oc1cccc2c(O)cccc12</smiles><smiles>[R]C(=C)COc1cccc2c(OCC([R])=C)cccc12</smiles>

(ii)
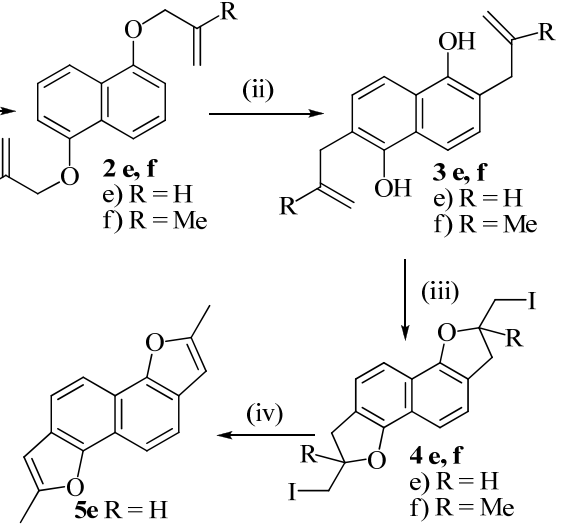

Scheme 3: Reagents and conditions: (i) Allyl bromide or 3-chloro-2-methyl-1-propene, acetone, $\mathrm{K}_{2} \mathrm{CO}_{3}$, NaI, reflux, 12 - 15 h; (ii) o-DCB, reflux, 8 - 10 h; (iii) Condition A: substrates 3e,f (1.0 mmol), NIS (2.0 mmol), MeCN, $0^{\circ} \mathrm{C}$-r.t, $4 \mathrm{~h}$; Condition B: substrates 3e,f (0.5 mmol), $I_{2}$ (1.5 equiv.), CTAB (0.30 mmol), $\mathrm{H}_{2} \mathrm{O}$ (10 ml.), r.t, $8 \mathrm{~h}$; (iv) $\mathrm{KOH} \mathrm{(2.0}$ mmol), EtOH, reflux, 2 - 3 h.

cyclized product in aqueous medium failed by using even upto 1.5 equivalents of molecular iodine for $24 \mathrm{~h}$ at room temperature. One of the problems encountered in such a reaction in aqueous media was the insolubility of the di-allyldihydroxy naphthalene precursors in water.

From the green chemistry point of view of water is the best solvent of choice as it is environment friendly and also very easily available. It is found that the reactivity of organic reaction in water media can be greatly enhanced by using surfactants $[46,47]$ which actually forms micelle and thus acts as a phase transfer catalyst in water medium. With these facts in our mind we have decided to carry out the iodocyclization of a series of di-allyldi- hydroxy naphthalene precursors 3a-f in the presence of the surfactant CTAB. So, the precursor 1,4-diallylnaphthalene-2,3-diol $3 \mathbf{a}(0.5 \mathrm{mmol})$ was treated with surfacetant CTAB $(0.30 \mathrm{mmol})$ and molecular iodine (1.5 equivalent) in water $(10 \mathrm{ml})$ for $8 \mathrm{~h}$ at room temperature. The aforesaid reaction condition (optimized condition) was arrived at by gradually varying either the concentration of iodine or surfactant and also by altering the reaction time (Table 1).

It is important to note that the same spot of $4 a\left(R_{f} 0.45\right.$ in petroleum ether: ethyl acetate 9:1) in TLC was formed by molecular iodine-mediated cyclization in the presence of surfactant $\mathrm{CTAB}$ in aqueous medium and as also in the $N$-iodosuccinimide-mediated reaction. After usual work up and silica gel column chromatrography a solid product was obtained. This was identified as $\mathbf{4 a}$ by comparison of spectroscopic data, melting point, mixed melting point and super imposable IR spectroscopy with the product 4a obtained by the other method. The reaction was then performed with other precursors $(\mathbf{3 b}-\mathbf{f})$ to give the products (4b-f) (Schemes 1-3).

The comparative results of two methodologies are summarized in Table 2.

The mechanism for the $N$-iodosuccinimide mediated cyclization and molecular iodine-mediated cyclization in water in the presence of $\mathrm{CTAB}$ as surfactant is somewhat similar and follows the electrophilic cyclization pathway.

Table 1. Optimized reaction conditions for molecular iodine-mediated cyclization of $3 a$ in aqueous micelle using CTAB as surfactant.

\begin{tabular}{ccccccc}
\hline Entry & $\begin{array}{c}\text { Substrate } \\
\text { (3a) mmol }\end{array}$ & $\begin{array}{c}\text { Surfactant } \\
\text { (CTAB) } \\
\text { mmol }\end{array}$ & $\begin{array}{c}\text { Iodine } \\
\text { equiv. }\end{array}$ & $\begin{array}{c}\text { Water } \\
\text { ml. }\end{array}$ & $\begin{array}{c}\text { Time } \\
(\mathrm{h})\end{array}$ & $\begin{array}{c}\text { Yield (\%) } \\
\text { of product } \\
(\mathbf{4 a})\end{array}$ \\
\hline 1 & 0.5 & 0.10 & 1.0 & 10 & 5 & 48 \\
2 & 0.5 & 0.15 & 1.0 & 10 & 8 & 52 \\
3 & 0.5 & 0.20 & 1.0 & 10 & 8 & 56 \\
4 & 0.5 & 0.20 & 1.5 & 10 & 8 & 65 \\
5 & 0.5 & 0.30 & 1.5 & 10 & 8 & 80 \\
6 & 0.5 & 0.50 & 1.5 & 10 & 10 & 80 \\
\hline
\end{tabular}

Table 2. Summarized results of the cyclization strategy.

\begin{tabular}{|c|c|c|c|c|}
\hline Entry & Substrate & Product & $\begin{array}{c}\text { Yield }(\%)^{\mathrm{a}} \\
(\mathbf{A})^{\mathrm{b}}\end{array}$ & $\begin{array}{l}\text { Yield }(\%)^{\mathrm{a}} \\
(\mathbf{B})^{\mathrm{b}}\end{array}$ \\
\hline 1 & $3 a$ & $4 a$ & 75 & 80 \\
\hline 2 & $3 \mathbf{b}$ & $4 b$ & 72 & 78 \\
\hline 3 & $3 c$ & $4 c$ & 80 & 85 \\
\hline 4 & 3d & 4d & 71 & 76 \\
\hline 5 & $3 \mathbf{e}$ & $4 e$ & 75 & 88 \\
\hline 6 & $3 f$ & 4f & 72 & 84 \\
\hline
\end{tabular}

Condition A: substrates 3a-3f $(1.0 \mathrm{mmol})$, NIS $(2.0 \mathrm{mmol}), \mathrm{MeCN}, 0^{\circ} \mathrm{C}$-r.t, $4 \mathrm{~h}$; condition B: substrates 3a-3f $(0.5 \mathrm{mmol}), \mathrm{I}_{2}(1.5$ equiv. $)$, CTAB $(0.30$ $\mathrm{mmol}), \mathrm{H}_{2} \mathrm{O}(10 \mathrm{ml})$, r.t, $8 \mathrm{~h} .{ }^{\mathrm{a}}$ Isolated yields are reported; ${ }^{\mathrm{b}}$ All reactions were performed under the optimized reaction conditions. 
The reaction of solid reagent in water takes place on the surface of water where collision of reacting partners is restricted. However, in the presence of a surfactant e.g. CTAB, the surface tension of water is lowered and allow easier spreading and lowering of interfacial tension between the two reacting partners. Surfactants are usually organic molecules containing both hydrophobic group (lipophilic tails called micelle) and hydrophilic groups (heads), are known to facilitate a number of reactions in aqueous medium. A micelle in a surfactant sticks to the reactant and due to its heavy head settles down into water from the surface and thus increases the collision between the two reacting partners in the intramolecular cyclization and results in more favourable conditions for the reaction compared to reaction on water surface $[46,47]$. At first the di-allyldi-hydroxy naphthalene precursor 3a may generate the iodonium intermediate $\mathbf{3 a}$, which undergoes 5-exo-trig cyclization to from the cyclized product 4a. The alternative 6-endo-trig pathway to produce the product 6a is not favoured and thus ruled out. The same mechanism may be followed in case of other sub-

\section{strates (Scheme 4).}

\section{Conclusions}

We have demonstrated that molecular iodine-mediated 5-exo-trig cyclization in aqueous micelle using CTAB as surfactant and $\mathrm{N}$-iodosuccinimide in actonitrile are efficacious strategies for the synthesis of a range of doubly fused $[5,6]$ heterocyclic compounds from a series of bis-allylhydroxy naphthalene precursors. The methodologies described here may be attractive for the synthesis of analogs of some important potentially bioactive heterocycles.

General. All the reagents were obtained from commercial sources and were used as such. Except methanol (which was HPLC-grade), the rest of the solvents were dried and distilled before use. Elemental analyses and Mass spectra (ESI+) were performed at the Indian Institute of Chemical Biology, Kolkata. IR spectra were recorded on $\mathrm{KBr}$ discs on a Perkin Elmer L 120-000A apparatus $\left(v_{\max }\right.$ in $\left.\mathrm{cm}^{-1}\right)$. Routine ${ }^{1} \mathrm{H}$ and ${ }^{13} \mathrm{C}\left\{{ }^{1} \mathrm{H}\right\}$ NMR

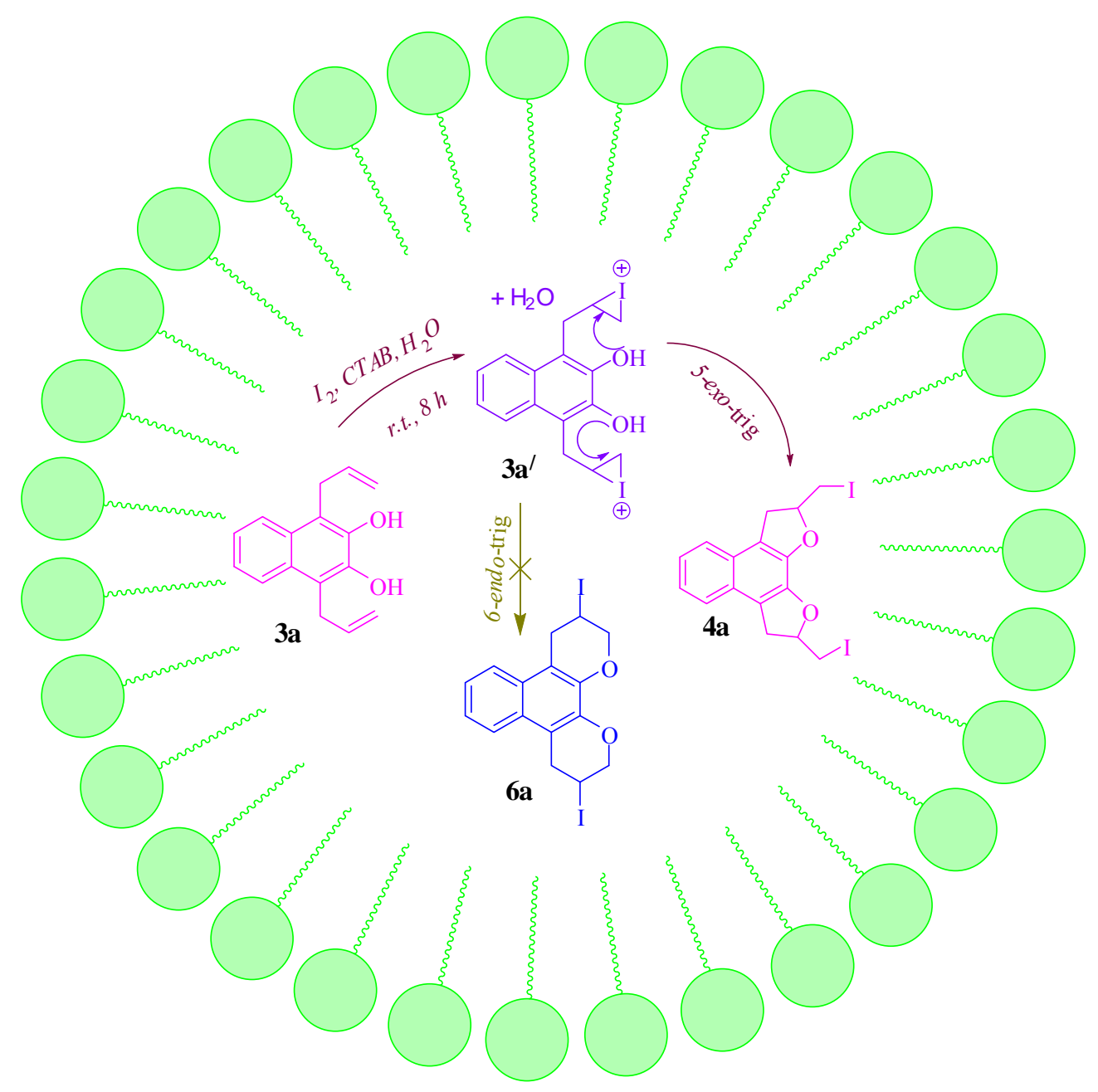

Scheme 4. Probable mechanistic pathway for the formation of 4 a. 
spectra were recorded with a Bruker DPX-400 MHz instrument at $298 \mathrm{~K}$. The chemical shifts $(\delta)$ were given in ppm and the coupling constants $(J)$ in $\mathrm{Hz} . \mathrm{CDCl}_{3}$ were used as solvent and $\mathrm{SiMe}_{4}$ as the reference standard for recording of spectra. Silica gel [(100 - 200, $230-400$ mesh), SRL, India] was used for chromatographic separation. Silica gel G [E-Marck (India)] and Silica gel 60F 254 [E-Marck (Germany)] were used for TLC. Petroleum ether refers to the fraction boiling between $60^{\circ} \mathrm{C}$ and $80^{\circ} \mathrm{C}$.

General procedure for the preparation of 2,3-bis (2-methylallyloxy) naphthalene (2b), 2,7-bis (2-methylallyloxy) naphthalene (2d), and 1,5-bis (2-methyllallyloxy) naphthalene (2f).

A mixture of the compound $\mathbf{1 b}$ or $\mathbf{1 d}$ or $\mathbf{1 f}(1 \mathrm{mmol})$, 3-chloro-2-methyl-1-propene $(2.5 \mathrm{mmol})$ and anhydrous $\mathrm{K}_{2} \mathrm{CO}_{3}(4.5 \mathrm{~g})$, NaI $(100 \mathrm{mg})$ were refluxed in anhydrous acetone $(75 \mathrm{~mL})$ for $12-15 \mathrm{~h}$. The mixture was cooled, filtered, and the solvent was evaporated. The residual mass was extracted with $\mathrm{CH}_{2} \mathrm{Cl}_{2}(2 \times 25 \mathrm{~mL})$, washed with water $(2 \times 25 \mathrm{~mL})$, and dried $\left(\mathrm{Na}_{2} \mathrm{SO}_{4}\right)$. The solvent $\left(\mathrm{CH}_{2} \mathrm{Cl}_{2}\right)$ was removed and the residual gummy mass was subjected to column chromatography over silica gel using petroleum ether-ethyl acetate $(9: 1)$ as eluent to produce the desired diallyl derivatives $\mathbf{2 b}$ or $\mathbf{2 d}$ or $\mathbf{2 f}$.

The simple diallyl derivatives $\mathbf{2 a}, \mathbf{2 c}$ and $\mathbf{2 e}$ were synthesized according to the literature procedure $[24,25]$.

2b: Reddish yellow solid, $82 \%$ yield; $\mathrm{mp} 46^{\circ} \mathrm{C}-48^{\circ} \mathrm{C}$; LRMS (70 eV, EI): $m / z=268\left(\mathrm{M}^{+}\right)$; IR (KBr): $v / \mathrm{cm}^{-1}=$ 3055, 2916, 1587, 1513, 1482, 1262, 1171, 1112, 1003, 857,$745 ;{ }^{1} \mathrm{H}$ NMR $\left(400 \mathrm{MHz}, \mathrm{CDCl}_{3}\right): \delta=1.86(\mathrm{~s}, 6 \mathrm{H}$, $\left.\mathrm{CH}_{3}\right), 4.60\left(\mathrm{~s}, 4 \mathrm{H}, \mathrm{OCH}_{2}\right), 5.05\left(\mathrm{~s}, 2 \mathrm{H},=\mathrm{CH}_{2}\right), 5.13(\mathrm{~s}$, $\left.2 \mathrm{H},=\mathrm{CH}_{2}\right), 7.10(\mathrm{~s}, 2 \mathrm{H}, \mathrm{ArH}), 7.28-7.33(\mathrm{~m}, 4 \mathrm{H}, \mathrm{ArH})$. Analysis Calcd for $\mathrm{C}_{18} \mathrm{H}_{20} \mathrm{O}_{2}$ (268.15): C, 80.56; H, $7.51 \%$. Found: C, 80.49, H, 7.58\%.

2d: Yellow solid, $74 \%$ yield; mp $166^{\circ} \mathrm{C}-168^{\circ} \mathrm{C}$; HRMS (EI): $m / z=268.1469\left(\mathrm{M}^{+}\right)$; IR (KBr): $v / \mathrm{cm}^{-1}=$ 3031, 2925, 1625, 1606, 1551, 1453, 1210, 1169, 1021, 904, 835; ${ }^{1} \mathrm{H}$ NMR $\left(300 \mathrm{MHz}, \mathrm{CDCl}_{3}\right): \delta=1.86(\mathrm{~s}, 6 \mathrm{H}$, $\left.\mathrm{CH}_{3}\right), 4.52\left(\mathrm{~s}, 4 \mathrm{H}, \mathrm{OCH}_{2}\right), 5.01\left(\mathrm{~s}, 2 \mathrm{H},=\mathrm{CH}_{2}\right), 5.15(\mathrm{~s}$, $\left.2 \mathrm{H},=\mathrm{CH}_{2}\right), 7.00-7.04(\mathrm{~m}, 4 \mathrm{H}, \mathrm{ArH}), 7.65(\mathrm{~d}, J 7.8 \mathrm{~Hz}$, $2 \mathrm{H}, \mathrm{ArH}),{ }^{13} \mathrm{C}$ NMR $\left(100 \mathrm{MHz}, \mathrm{CDCl}_{3}\right): \delta=19.4,71.7$, 106.6, 112.2, 116.4, 124.4, 129.1, 135.8, 140.9, 157.3. Analysis Calcd for $\mathrm{C}_{18} \mathrm{H}_{20} \mathrm{O}_{2}$ (268.15): C, 80.56; H, $7.51 \%$. Found: C, 80.64, H, 7.62\%.

2f: Yellow solid, $78 \%$ yield; $\mathrm{mp} 86^{\circ} \mathrm{C}-88^{\circ} \mathrm{C}$; LRMS (70 eV, EI): $m / z=268\left(\mathrm{M}^{+}\right)$; IR $(\mathrm{KBr}): v / \mathrm{cm}^{-1}=3076$, $2976,1655,1592,1512,1418,1264,1043,904,774 ;{ }^{1} \mathrm{H}$ NMR $\left(300 \mathrm{MHz}, \mathrm{CDCl}_{3}\right): \delta=1.91\left(\mathrm{~s}, 6 \mathrm{H}, \mathrm{CH}_{3}\right), 4.59(\mathrm{~s}$, $\left.4 \mathrm{H}, \mathrm{OCH}_{2}\right), 5.03\left(\mathrm{~s}, 2 \mathrm{H},=\mathrm{CH}_{2}\right), 5.20\left(\mathrm{~s}, 2 \mathrm{H},=\mathrm{CH}_{2}\right), 6.81$ (d, $J 7.8 \mathrm{~Hz}, 2 \mathrm{H}, \mathrm{ArH}), 7.37$ (t, $J .8 \mathrm{~Hz}, 2 \mathrm{H}, \mathrm{ArH}), 7.91$ (d, J 7.8 Hz, $2 \mathrm{H}, \mathrm{ArH}$ ). Analysis Calcd for $\mathrm{C}_{18} \mathrm{H}_{20} \mathrm{O}_{2}$ (268.15): C, 80.56; H, 7.51\%. Found: C, 80.41, H, $7.38 \%$.
General procedure for the preparation of precursors: 1,4-bis(2-methylallyl) naphthalene -2,3-diol (3b), or 1,8-bis(2-methylallyl) naphthalene-2,7-diol (3d), or 2,6-bis (2-methylallyl)naphthalene-1,5-diol (3f):

Compound $\mathbf{2 b}$ or $\mathbf{2 d}$ or $\mathbf{2 f}$ was refluxed in $o$-dichloro benzene $(5 \mathrm{~mL})$ for about $8-10 \mathrm{~h}$. The mixture was cooled and directly subjected to column chromatography over solid silica gel. The desired product (3b or 3d or 3f) was obtained in pure form after usual column chromatographic separation using petroleum ether-ethyl acetate $(8: 2)$ as eluent.

The other bis-allylhydroxy naphthalene derivatives $\mathbf{3 a}$, 3c and 3e were synthesized according to the literature procedure [24,25].

3b: White solid, $71 \%$ yield; $\mathrm{mp} 120^{\circ} \mathrm{C}-121^{\circ} \mathrm{C}$; LRMS (70 eV, EI): $m / z=268\left(\mathrm{M}^{+}\right)$; IR $(\mathrm{KBr}): v / \mathrm{cm}^{-1}=3377$, 1628, 1607, 1517, 1454, 1384, 1355, 1259, 1221, 1031, 966, 845, 742; ${ }^{1} \mathrm{H}$ NMR $\left(400 \mathrm{MHz}, \mathrm{CDCl}_{3}\right): \delta=1.84(\mathrm{~s}$, $\left.6 \mathrm{H}, \mathrm{CH}_{3}\right), 3.80\left(\mathrm{~s}, 4 \mathrm{H}, \mathrm{CH}_{2}\right), 4.72\left(\mathrm{~s}, 2 \mathrm{H},=\mathrm{CH}_{2}\right), 4.90(\mathrm{~s}$, $\left.2 \mathrm{H},=\mathrm{CH}_{2}\right), 5.83(\mathrm{~s}, 2 \mathrm{H}, \mathrm{OH}), 7.30-7.36(\mathrm{~m}, 2 \mathrm{H}, \mathrm{ArH})$, $7.66(\mathrm{~d}, J 7.2 \mathrm{~Hz}, 1 \mathrm{H}, \mathrm{ArH}), 7.82(\mathrm{~d}, J 7.2 \mathrm{~Hz}, 1 \mathrm{H}, \mathrm{ArH})$; ${ }^{13} \mathrm{C}$ NMR $\left(100 \mathrm{MHz}, \mathrm{CDCl}_{3}\right): \delta=22.7,34.1,109.0$, $112.0,118.2,121.9,123.3,124.2,127.3,128.8,129.9$, 142.9, 144.3. Analysis Calcd for $\mathrm{C}_{18} \mathrm{H}_{20} \mathrm{O}_{2}$ (268.15): C, 80.56; H, 7.51\%. Found: C, 80.47, H, 7.68\%.

3d: Reddish yellow solid, $76 \%$ yield; $\mathrm{mp} 90^{\circ} \mathrm{C}-92^{\circ} \mathrm{C}$; LRMS (70 eV, EI): $m / z=268\left(\mathrm{M}^{+}\right) ; \mathrm{IR}(\mathrm{KBr}): \mathrm{v} / \mathrm{cm}^{-1}=$ 3407, 2973, 1627, 1516, 1456, 1355, 1280, 1207, 1018, 833; ${ }^{1} \mathrm{H}$ NMR $\left(400 \mathrm{MHz}, \mathrm{CDCl}_{3}\right): \delta=1.86\left(\mathrm{~s}, 6 \mathrm{H}, \mathrm{CH}_{3}\right)$, $4.51\left(\mathrm{~s}, 4 \mathrm{H}, \mathrm{CH}_{2}\right), 5.01\left(\mathrm{~s}, 2 \mathrm{H},=\mathrm{CH}_{2}\right), 5.14(\mathrm{~s}, 2 \mathrm{H}$, $\left.=\mathrm{CH}_{2}\right), 5.34(\mathrm{~s}, 2 \mathrm{H}, \mathrm{OH}), 6.91-7.03(\mathrm{~m}, 2 \mathrm{H}, \mathrm{ArH}), 7.65$ (d, $J 8.8 \mathrm{~Hz}, 2 \mathrm{H}, \mathrm{ArH})$. Analysis Calcd for $\mathrm{C}_{18} \mathrm{H}_{20} \mathrm{O}_{2}$ (268.15): C, 80.56; H, 7.51\%. Found: C, 80.72, H, $7.60 \%$.

3f: Yellow gummy mass, $74 \%$ yield; LRMS $(70 \mathrm{eV}$, $\mathrm{EI}): m / z=268\left(\mathrm{M}^{+}\right)$; IR $(\mathrm{KBr}): v / \mathrm{cm}^{-1}=3481,2917$, 1653, 1509, 1408, 1257, 1072, 1048, 898, 794; ${ }^{1} \mathrm{H}$ NMR $\left(400 \mathrm{MHz}, \mathrm{CDCl}_{3}\right): \delta=1.76\left(\mathrm{~s}, 6 \mathrm{H}, \mathrm{CH}_{3}\right), 3.44(\mathrm{~s}, 2 \mathrm{H}$, $\left.\mathrm{CH}_{2}\right), 3.54\left(\mathrm{~s}, 2 \mathrm{H}, \mathrm{CH}_{2}\right), 4.71\left(\mathrm{~s}, 2 \mathrm{H},=\mathrm{CH}_{2}\right), 4.92(\mathrm{~s}, 2 \mathrm{H}$, $\left.=\mathrm{CH}_{2}\right), 5.76(\mathrm{~s}, 2 \mathrm{H}, \mathrm{OH}), 7.76(\mathrm{~d}, J 8.4 \mathrm{~Hz}, 2 \mathrm{H}, \mathrm{ArH})$, $7.86(\mathrm{~d}, J 8.4 \mathrm{~Hz}, 2 \mathrm{H}, \mathrm{ArH}) ;{ }^{13} \mathrm{C}$ NMR $(100 \mathrm{MHz}$, $\left.\mathrm{CDCl}_{3}\right): \delta=22.4,37.2,112.5,114.6,118.4,125.2,128.1$, 143.0, 149.9. Analysis Calcd for $\mathrm{C}_{18} \mathrm{H}_{20} \mathrm{O}_{2}$ (268.15): C, 80.56 ; H, 7.51\%. Found: C, 80.42, H, 7.34\%.

General procedure for the preparation of $4(a-f)$ using $N$-Iodosuccinimide: (Condition A).

$\mathrm{N}$-iodosuccinimide $(0.450 \mathrm{~g}, 2 \mathrm{mmol})$ was added to a dry and distilled acetonitrile $(20 \mathrm{~mL})$ solution of the compounds $3(\mathbf{a}-\mathbf{f})(1 \mathrm{mmol})$ at $0^{\circ} \mathrm{C}-5^{\circ} \mathrm{C}$ under nitrogen atmosphere. The reaction mixture was then magnetically stirred for $1 \mathrm{~h}$ at $0^{\circ} \mathrm{C}-5^{\circ} \mathrm{C}$. It was then stirred for an additional period of $3 \mathrm{~h}$ at room temperature. On completion of the reaction (as monitored by TLC), acetonitrile was removed from the reaction mixture under reduced pres- 
sure. The residual mass was then extracted with $\mathrm{CH}_{2} \mathrm{Cl}_{2}$ $(3 \times 25 \mathrm{~mL})$ and was washed with $5 \% \mathrm{NaHSO}_{3}$ solution $(3 \times 25 \mathrm{~mL})$ and water $(3 \times 25 \mathrm{~mL})$ and dried $\left(\mathrm{Na}_{2} \mathrm{SO}_{4}\right)$. The residual mass after removal of the solvent $\left(\mathrm{CH}_{2} \mathrm{Cl}_{2}\right)$ was subjected to column chromatography over silica gel using petroleum ether-ethyl acetate $(9: 1)$ as eluent to give the products $\mathbf{4}(\mathbf{a}-\mathbf{f})$ in $71 \%$ - $80 \%$ yields.

General procedure for the molecular iodine-mediated cyclization of $3(a-f)$ in aqueous medium in the presence of CTAB: (Condition B).

Di-allyl-dihydroxy naphthalene derivatives 3(a-f) (0.5 mmol), surfactant CTAB (122 $\mathrm{mg}, 0.30 \mathrm{mmol})$ and water $(10 \mathrm{~mL})$ were mixed in a conical flask $(50 \mathrm{~mL})$, and the mixture was stirred at $0^{\circ} \mathrm{C}-5^{\circ} \mathrm{C}$ for $15 \mathrm{~min}$. Molecular iodine (192 mg, 1.5 equivalent) was added in three portions at an interval of $10-15$ minutes and the reaction mixture was stirred at $0^{\circ} \mathrm{C}-5^{\circ} \mathrm{C}$ for $1 \mathrm{~h}$ and then at r.t. for $7 \mathrm{~h}$. After the reaction was over (as detected by TLC), the reaction mixture was extracted with EtOAc $(3 \times 15 \mathrm{~mL})$, washed with saturated sodium sulphite solution $(2 \times 10$ $\mathrm{mL})$, and brine $(1 \times 10 \mathrm{~mL})$, dried over anhydrous sodium sulphate. The solvent was distilled off in a rotary evaporator at r.t. under reduced pressure. The crude mass was purified by chromatography over silica gel using petroleum ether-ethyl acetate $(9: 1)$ as eluent to give the pure products $4(\mathbf{a}-\mathbf{f})$ in $76 \%-88 \%$ yields.

4a: White solid, mp $197^{\circ} \mathrm{C}-199^{\circ} \mathrm{C}$; HRMS (EI): $\mathrm{m} / \mathrm{z}=$ $491.9088\left(\mathrm{M}^{+}\right), 514.8431(\mathrm{M}+\mathrm{Na})^{+}$. IR $(\mathrm{KBr}): v / \mathrm{cm}^{-1}=$ 3036, 2938, 2918, 1633, 1596, 1526, 1460, 1377, 1285, 1180, 1119, 1027, 982, 951, 855, 744, 622; ${ }^{1} \mathrm{H}$ NMR $\left(400 \mathrm{MHz}, \mathrm{CDCl}_{3}\right): \delta=3.37(\mathrm{dd}, J 15.2,6.8 \mathrm{~Hz}, 2 \mathrm{H}$, $\left.\mathrm{CH}_{2}\right), 3.39-3.43\left(\mathrm{~m}, 2 \mathrm{H}, \mathrm{CH}_{2}\right), 3.54-3.60\left(\mathrm{~m}, 2 \mathrm{H}, \mathrm{CH}_{2}\right)$, $3.68\left(\mathrm{dd}, J 15.6,9.6 \mathrm{~Hz}, 2 \mathrm{H}, \mathrm{CH}_{2}\right), 5.12-5.18(\mathrm{~m}, 2 \mathrm{H}$, $\mathrm{CH}), 7.37$ (dd, J 6.0, $3.2 \mathrm{~Hz}, 2 \mathrm{H}, \mathrm{ArH}), 7.58$ (dd, J 6.0, $3.2 \mathrm{~Hz}, 2 \mathrm{H}, \mathrm{ArH}) ;{ }^{13} \mathrm{C} \mathrm{NMR}\left(100 \mathrm{MHz}, \mathrm{CDCl}_{3}\right): \delta=8.9$, 35.4, 83.6, 119.6, 123.5, 124.1, 126.4, 143.3. Analysis Calcd for $\mathrm{C}_{16} \mathrm{H}_{14} \mathrm{I}_{2} \mathrm{O}_{2}$ (491.91): C, 39.05, H, 2.87\%. Found: C, 39.19, H, 3.01\%.

4b: Yellow solid, mp $106^{\circ} \mathrm{C}-108^{\circ} \mathrm{C}$; LRMS $(70 \mathrm{eV}$, $\mathrm{EI}): m / z=520\left(\mathrm{M}^{+}\right) ; \mathrm{IR}(\mathrm{KBr}): v / \mathrm{cm}^{-1}=3019,1638$, 1606, 1520, 1464, 1385, 1295, 1138, 1055, 965, 893, 761; ${ }^{1} \mathrm{H}$ NMR (300 MHz, $\left.\mathrm{CDCl}_{3}\right): \delta=1.76\left(\mathrm{~s}, 6 \mathrm{H}, \mathrm{CH}_{3}\right), 3.36$ (d, $\left.J 15.6 \mathrm{~Hz}, 2 \mathrm{H}, \mathrm{CH}_{2}\right), 3.52\left(\mathrm{~s}, 4 \mathrm{H}, \mathrm{CH}_{2}\right), 3.61$ (d, $J$ 15.6 Hz, 2H, $\mathrm{CH}_{2}$ ), 7.24-7.37 (m, 1H, ArH), 7.50 (dd, $J$ 8.4, 0.9 Hz, 2H, ArH), 7.67 (dd, $J 8.4,1.5 \mathrm{~Hz}, 1 \mathrm{H}, \mathrm{ArH})$; ${ }^{13} \mathrm{C}$ NMR (100 MHz, $\left.\mathrm{CDCl}_{3}\right): \delta=15.2,26.4,40.9,89.0$, 110.6, 123.7, 124.2, 127.3, 141.3. Analysis Calcd for $\mathrm{C}_{18} \mathrm{H}_{18} \mathrm{I}_{2} \mathrm{O}_{2}$ (519.94): C, 41.56, H, 3.49\%. Found: $\mathrm{C}$, 41.42, H, 3.31\%.

4c: Reddish yellow gummy mass; LRMS (70 eV, EI): $m / z=492\left(\mathrm{M}^{+}\right)$; IR $(\mathrm{KBr}): \mathrm{v} / \mathrm{cm}^{-1}=3018,1632,1518$, $1457,1373,1241,1218,1061,994,933,827,772 ;{ }^{1} \mathrm{H}$ NMR (400 MHz, $\mathrm{CDCl}_{3}$ ): $\delta=3.19-3.27\left(\mathrm{~m}, 2 \mathrm{H}, \mathrm{CH}_{2}\right.$ ), 3.37-3.47 (m, 2H, $\left.\mathrm{CH}_{2}\right), 3.48$ - 3.53 (m, 2H), 3.62 (dd, $J$
15.6, 6.4 Hz, 2H, $\left.\mathrm{CH}_{2}\right)$, 5.04-5.07 (m, 2H, $\left.\mathrm{CH}\right)$, 7.58-7.63 (m, 2H, ArH), 7.71 (dd, $J 11.6,8 \mathrm{~Hz}, 2 \mathrm{H}, \mathrm{ArH})$; ${ }^{13} \mathrm{C}$ NMR $\left(100 \mathrm{MHz} \mathrm{CDCl}_{3}\right): \delta=9.3,39.9,81.9,109.4$, 117.8, 124.8, 129.0, 132.7, 157.3. Analysis Calcd for $\mathrm{C}_{16} \mathrm{H}_{14} \mathrm{I}_{2} \mathrm{O}_{2}$ (491.91): C, 39.05, H, 2.87\%. Found: C, $39.22, \mathrm{H}, 2.99 \%$.

4d: Grey gummy mass; LRMS (70 eV, EI): $m / z=520$ $\left(\mathrm{M}^{+}\right)$; IR $(\mathrm{KBr}): v / \mathrm{cm}^{-1}=3076,2974,1626,1512,1451$, 1377, 1275, 1195, 1053, 971, 903, 829; ${ }^{1} \mathrm{H}$ NMR (400 $\left.\mathrm{MHz}, \mathrm{CDCl}_{3}\right): \delta=1.89\left(\mathrm{~s}, 6 \mathrm{H}, \mathrm{CH}_{3}\right), 4.61\left(\mathrm{~s}, 4 \mathrm{H}, \mathrm{CH}_{2}\right)$, $5.06\left(\mathrm{~s}, 2 \mathrm{H}, \mathrm{CH}_{2}\right), 5.21\left(\mathrm{~s}, 2 \mathrm{H}, \mathrm{CH}_{2}\right), 7.10(\mathrm{~d}, J 8.8 \mathrm{~Hz}$, 2H, ArH), 7.65 (d, $J 8.8 \mathrm{~Hz}, 2 \mathrm{H}, \mathrm{ArH}) ;{ }^{13} \mathrm{C}$ NMR $(100$ $\left.\mathrm{MHz}, \mathrm{CDCl}_{3}\right): \delta=19.5,26.2,42.1,85.3,113.9,116.7$, 124.8, 130.2, 136.3, 158.8. Analysis Calcd for $\mathrm{C}_{18} \mathrm{H}_{18} \mathrm{I}_{2} \mathrm{O}_{2}$ (519.94): C, 41.56, H, 3.49\%. Found: $\mathrm{C}$, 41.38, H, 3.58\%.

4e: White solid, $\mathrm{mp} 168^{\circ} \mathrm{C}-170^{\circ} \mathrm{C}$; HRMS (EI): $m / z=$ $491.9078\left(\mathrm{M}^{+}\right)$, IR $(\mathrm{KBr}): v / \mathrm{cm}^{-1}=3414,2899,1599$, $1527,1400,1284,1218,1174,1115,1067,966,804 ;{ }^{1} \mathrm{H}$ NMR (400 MHz, $\left.\mathrm{CDCl}_{3}\right): \delta=3.21(\mathrm{dd}, J 15.6,6.4 \mathrm{~Hz}$, $\left.2 \mathrm{H}, \mathrm{CH}_{2}\right), 3.36-3.41\left(\mathrm{~m}, 2 \mathrm{H}, \mathrm{CH}_{2}\right), 3.51-3.58(\mathrm{~m}, 4 \mathrm{H}$, $\left.\mathrm{CH}_{2}\right), 5.03-5.10(\mathrm{~m}, 2 \mathrm{H}, \mathrm{CH}), 7.28(\mathrm{~d}, J 8.4 \mathrm{~Hz}, 2 \mathrm{H}$, ArH), 7.49 (d, J 8.0 Hz, 2H, ArH); ${ }^{13} \mathrm{C}$ NMR (100 MHz, $\left.\mathrm{CDCl}_{3}\right): \delta=9.3,36.9,82.1,114.2,118.7,120.9,122.4$, 154.5. Analysis Calcd for $\mathrm{C}_{16} \mathrm{H}_{14} \mathrm{I}_{2} \mathrm{O}_{2}$ (491.91): C, 39.05, H, $2.87 \%$. Found: C, 38.92, H, 2.66\%.

4f: Light yellow solid, mp $72^{\circ} \mathrm{C}-74^{\circ} \mathrm{C}$; LRMS $(70 \mathrm{eV}$, $\mathrm{EI}): m / z=520\left(\mathrm{M}^{+}\right)$; IR $(\mathrm{KBr}): v / \mathrm{cm}^{-1}=3013,2971$, 2914, 1596, 1518, 1407, 1292, 1162, 1031, 996, 909, 787; ${ }^{1} \mathrm{H}$ NMR (400 MHz, $\left.\mathrm{CDCl}_{3}\right): \delta=1.91\left(\mathrm{~s}, 6 \mathrm{H}, \mathrm{CH}_{3}\right), 3.24$ (d, J $\left.15.6 \mathrm{~Hz}, 2 \mathrm{H}, \mathrm{CH}_{2}\right), 3.45-3.53\left(\mathrm{~m}, 6 \mathrm{H}, \mathrm{CH}_{2}\right), 7.53$ (d, J $8.4 \mathrm{~Hz}, 2 \mathrm{H}, \mathrm{ArH}), 7.86$ (d, J $8.4 \mathrm{~Hz}, 2 \mathrm{H}, \mathrm{ArH}) ;{ }^{13} \mathrm{C}$ NMR $\left(100 \mathrm{MHz}, \mathrm{CDCl}_{3}\right): \delta=19.6,26.3,42.1,87.2$, $113.9,122.1,125.4,126.2,154.5$. Analysis Calcd for $\mathrm{C}_{18} \mathrm{H}_{18} \mathrm{I}_{2} \mathrm{O}_{2}$ (519.94): C, 41.56, H, 3.49\%. Found: C, 41.60, H, 3.34\%.

General procedure for the dehydroiodination of compounds 4a, 4c and 4e:

Compound $4 \mathbf{a}(1 \mathrm{mmol})$ was dissolved in rectified spirit $(5 \mathrm{~mL})$ and potassium hydroxide $(0.112 \mathrm{~g}, 2 \mathrm{mmol})$ was added and the reaction mixture was refluxed for 2 - 3 h. After the reaction was over (as detected by TLC), rectified spirit was removed and the residue was extracted with dichloromethane $(3 \times 15 \mathrm{~mL})$ and washed with water $(2 \times 20 \mathrm{~mL})$ and dried $\left(\mathrm{Na}_{2} \mathrm{SO}_{4}\right)$. The residual mass after removal of the solvent was subjected to column chromatography over silica gel using petroleum etherethyl-acetate $(9: 1)$ as eluent to give the product 5a (89\%).

Similarly the dehydroiodination of compounds $4 \mathbf{c}$ and 4e were carried out to give $\mathbf{5 c}$ and $5 \mathbf{e}$, respectively and found to have identical spectral data as that of literature report [24].

5a: Light yellow solid, $89 \%$ yield; mp. $146^{\circ} \mathrm{C}-147^{\circ} \mathrm{C}$; 
HRMS (EI): $m / z=237.0918(\mathrm{M}+\mathrm{H})^{+}$. IR (KBr): $v / \mathrm{cm}^{-1}$ $=2912,1625,1595,1434,1358,1192,926,809,754 ;{ }^{1} \mathrm{H}$ NMR (400 MHz, $\left.\mathrm{CDCl}_{3}\right): \delta=2.56\left(\mathrm{~s}, 6 \mathrm{H}, \mathrm{CH}_{3}\right), 6.86(\mathrm{~s}$, $2 \mathrm{H},=\mathrm{CH}), 7.50(\mathrm{dd}, J 6.0,3.2 \mathrm{~Hz}, 2 \mathrm{H}, \mathrm{ArH}), 8.07(\mathrm{dd}, J$ $6.0,3.2 \mathrm{~Hz}, 2 \mathrm{H}, \mathrm{ArH}) ;{ }^{13} \mathrm{C}$ NMR $\left(100 \mathrm{MHz}, \mathrm{CDCl}_{3}\right): \delta=$ 14.1, 102.5, 122.1, 124.0, 124.3, 124.7, 139.0, 153.6. Analysis Calcd for $\mathrm{C}_{16} \mathrm{H}_{12} \mathrm{O}_{2}$ (236.08): C, 81.34, H, $5.12 \%$. Found: C, 81.22 , H, 5.26\%.

\section{Acknowledgements}

We thank to UGC (New Delhi) for providing financial support [F. PSW-013/ 11 - 12 (ERO)]. One of us (PKB) dedicates this paper in the commemoration of his beloved late father, Sri Bharati Bhusan Basu. AG is grateful to DST (New Delhi) for a research fellowship (SRF). We are extremely grateful to Prof. K.C. Majumdar, University of Kalyani for generous help during the correction of the manuscript. We are thankful to Sri. Latibuddin Thander of our department for help and kind cooperation.

\section{REFERENCES}

[1] S. Vincent, S. Chen, D. B. Wilson and B. Ganem, "Probing the Overlap of Chorismate Mutase and Prephenate Dehydrogenase Sites in the escherichia coli T-Protein: A Dehydrogenase-Selective Inhibitor," Bioorganic \& Medicinal Chemistry Letters, Vol. 12, No. 6, 2002, pp. 929-931. doi:10.1016/S0960-894X(02)00053-7

[2] D. M. Schultz, J. A. Prescher, S. Kidd, D. Marona-Lewicka, D. E. Nichols and A. Monte, "'Hybrid' benzofuran-benzopyran congeners as rigid analogs of hallucinogenic phenethylamines," Bioorganic \& Medicinal Chemistry Letters, Vol. 16, No. 11, 2008, pp. 6242-6251.

doi:10.1016/j.bmc.2008.04.030

[3] C. S. Goksu, Uguz, M. T. Uguz, H. Ozdemir and H. A. Secen, "Concise Synthesis and the Antibacterial Activity of 5,6-Dimethoxy Naphthalene-2-Carboxylic Acid," Turkish Journal of Chemistry, Vol. 29, No. 2, 2005, pp. 199205.

[4] T. R. Burke, M. R. Fesen, A. Mazumder, J. Wang, A. M. Carothers, D. Grunberger, J. Driscoll, K. Kohn and Y. Pommier, "Hydroxylated Aromatic Inhibitors of HIV-1 Integrase," Journal of Medicinal Chemistry, Vol. 38, No. 21, 1995, pp. 4171-4178. doi:10.1021/jm00021a006

[5] T. R. Burke, B. Lim, V. E. Marquez, Z. H. Li, J. B. Bolen, I. Stefanova and I D. Horak, "Bicyclic Compounds as Ring-Constrained Inhibitors of Protein-Tyrosine Kinase p56lck," Journal of Medicinal Chemistry, Vol. 36, No. 4, 1993, pp. 425-432.doi:10.1021/jm00056a001

[6] K. Carl and L. U. S. Joseph, Patent 4,073,912, Chemical Abstracts, 19 78, 89, 24156, 1972.

[7] S. Rizzo, C. Riviere, L. Piazzi, A. Bisi, S. Gobbi, M. Bartolini, V. Andrisano, F. Morroni, A. Tarozzi, J.-P. Monti and A. Rampa, "Benzofuran-Based Hybrid Compounds for the Inhibition of Cholinesterase Activity, $\beta$ Amyloid Aggregation, and A $\beta$ Neurotoxicity," Journal of Medicinal Chemistry, Vol. 51, No. 10, 2008, pp. 2883-
2886. doi: $10.1021 / \mathrm{jm} 8002747$

[8] C. Kirilmis, M. Ahmedzade, S. Servi, M. Koca, A. Kizirgil and C. Kazaz, "Synthesis and antimicrobial activity of some novel derivatives of benzofuran: Part 2. The synthesis and antimicrobial activity of some novel 1-(1-benzofuran-2-yl)-2-mesitylethanone derivatives," European Journal of Medicinal Chemistry, Vol. 43, No. 2, 2008, pp. 300-308. doi:10.1016/j.ejmech.2007.03.023

[9] K. Ando, Y. Kawamura, Y. Akai, J. Kunitomo, T. Yokomizo, M. Yamashita, S. Ohta, T. Ohishi and Y. Ohishi, "Preparation of 2-,3-,4- and 7-(2-Alkylcarbamoyl-1-alkylvinyl)Benzo [b] Furans and Their BLT 1 and/or BLT Inhibitory Activities," Organic \& Biomolecular Chemistry, Vol. 6, No. 2, 2008, pp. 296-307. doi:10.1039/b710935k

[10] M. Ettaoussi, B. Peres, D. Klupsch, P. Delagrange, J.-A. Boutin, P. Renard, D.-H. Caignard, P. Chavatte, P. Berthelot, D. Lesieur and S. Yous, " Design and Synthesis of Benzofuranic Derivatives as New Ligands at the Melatonin-Binding Site $\mathrm{MT}_{3}$," Bioorganic \& Medicinal Chemistry, Vol. 16, No. 9, 2008, pp. 4954-4962. doi:10.1016/j.bmc.2008.03.036

[11] Y.-K. Lee, M.-H. Woo, C.-H. Kim, Y. Kim, S.-H. Lee, B.-S. Jeong, H.-W. Chang and J.-K. Son, "Two New Benzofurans from Gastrodia Elata and Their DNA Topoisomerases I and II Inhibitory Activities," Planta Medica, Vol. 73, No. 12, 2007, pp. 1287-1291.

doi:10.1055/s-2007-981619

[12] K. Oda, Y. Yamaguchi, T. Yoshimura, K. Wada and N. Nishizono, "Synthetic Models Related to Furanocoumarin-CYP 3A4 Interactions. Comparison of Furanocoumarin, Coumarin, and Benzofuran Dimers as Potent Inhibitors of CYP3A4 Activity," Chemical and Pharmaceutical Bulletin, Vol. 55, No. 9, 2007, pp.1419-1421. doi: $10.1248 / \mathrm{cpb} .55 .1419$

[13] G. F. Filzen, L. Bratton, X.-M. Cheng, N. Erasga, A. Geyer, C. Lee, G. Lu, J. Pulaski, R. J. Sorenson, P. C. Unangst, B. K. Trivedi and X. Xu, "Synthesis and SAR of Selective Benzothiophene, Benzofuran, and Indole-Based Peroxisome Proliferator-Activated Receptor $\delta$ Agonists," Bioorganic \& Medicinal Chemistry Letters, Vol. 17, No. 13, 2007, pp. 3630-635. doi:10.1016/j.bmcl.2007.04.047

[14] M. Useglio, P. M. Castellano, M. A. Operto, R. Torres and T. S. Kaufman, "Synthesis of $3 H$-Spiro-[benzofuran-2,1'-cyclohexane] Derivatives from Naturally Occurring Filifolinol and Their Classical Complement Pathway Inhibitory Activity," Bioorganic \& Medicinal Chemistry Letters, Vol. 16, No. 19, 2006, pp. 5097-5101. doi:10.1016/j.bmcl.2006.07.029

[15] H. J. Patel, J. Sarra, F. Caruso, M. Rossi, U. Doshi and R. A. Stephani, "Synthesis and Anticonvulsant Activity of New N-1',N-3'-Disubstituted-2' H,3H,5' H-Spiro-(2-benzofuran-1,4'-imidazolidine)-2',3,5'-triones," Bioorganic \& Medicinal Chemistry Letters, Vol. 16, No. 17, 2006, pp. 4644-4647. doi:10.1016/j.bmcl.2006.05.102

[16] J. S. Lazo, R. Nunes, J. J. Skoko, P. E. Q. de Oliveira, A. Vogt and P. Wipf, "Novel Benzofuran Inhibitors of Human Mitogen-Activated Protein Kinase Phosphatase-1," Bioorganic \& Medicinal Chemistry Letters, Vol. 14, No. 
16,2006, pp. $5643-5650$. doi:10.1016/j.bmc.2006.04.036

[17] K. C. Majumdar and S. Mondal, "A New Strategy For The Synthesis Of Coumarin- and Quinolone-Annulated Pyrroles via Pd(0)-Mediated Cross Coupling Followed by $\mathrm{Cu}(\mathrm{I})$-Catalyzed Heteroannulation," Tetrahedron Letters, Vol. 49, No. 15, 2008, pp. 2418-2420. doi:10.1016/j.tetlet.2008.02.044

[18] K. C. Majumdar, S. Mondal and N. De, "Synthesis of Cyclic Sulfonamides via Pd-Catalyzed Intramolecular Coupling Reaction: An Expedient Approach to Polycyclic Sultams," Synlett, No. 18, 2008, pp. 2851-2855. doi:10.1055/s-0028-1083502

[19] K. C. Majumdar, S. Mondal, D. Ghosh and B. Chattopadhyay, "Concise Synthesis of Pyrimido-azocine Derivatives via Aza-Claisen Rearrangement and Intramolecular Heck Reaction," Synthesis, Vol. 2010, No. 8, 2010, pp. 1315-1320.

[20] K. C. Majumdar, S. Mondal and D. Ghosh, "Concise Access to Pyrimidine-Annulated Azepine and Azocine Derivatives by Ru-Catalyzed Ring-Closing Metathesis," Synthesis, Vol. 2010, No., 2010, pp. 1176-1180.

[21] K. C. Majumdar, S. Mondal and D. Ghosh, "Synthesis of Tricyclic and Tetracyclicsultones by Pd-Catalysed Intramoleclar Cyclisation," Tetrahedron Letters, Vol. 50, No. 33, 2009, pp. 4781-4784. doi:10.1016/j.tetlet.2009.06.028

[22] K. C. Majumdar, S. Mondal and N. De, "Synthesis of Polycyclic Sultams by Pd-Catalyzed Intramolecular Cyclization," Synthesis, No. 18, 2009, pp. 3127-3135. doi:10.1055/s-0029-1216888

[23] K. C. Majumdar, S. Chakravorty and N. De, "Palladium-Mediated bis- and tris-biaryl Heck Coupling for the Synthesis of Heterocycles," Tetrahedron Letters, Vol. 49, No. 21, 2008, pp. 3419-3422. doi:10.1016/j.tetlet.2008.03.118

[24] K. C. Majumdar, B. Chattopadhyay and S. Chakravorty, "An Expedient Palladium-Mediated Intramolecular Cyclization Route to the Synthesis of Bis-Fused Benzofuran and a Two-Directional Ring Closing Metathesis for the Synthesis of Bis-Oxepin and Bis-Benzoxocine," Synthesis, Vol. 2009, pp. 674-680.

[25] B. J. Shorthill and T. E. Glass, "Naphthalene Based Calixarenes: Unusual Regiochemistry of a Friedel-Crafts Alkylation," Organic Letters, Vol. 3, No. 4, 2001, pp. 577579. doi:10.1021/o1006980+

[26] K. C. Majumdar, U. K. Kundu, U. Das, N. K. Jana and B. Roy, " $N$-Iodosuccinimide-An Effective Reagent for Regioselective Heterocyclization of $o$-cyclohex-2-enylanilines for the Synthesis of hexahydrocarbazoles," Canadian Journal of Chemistry, Vol. 83, No. 1, 2005, pp. 63-67. doi:10.1139/v04-162

[27] K. C. Majumdar, P. K. Basu and B. Roy, "N-Iodosuccinimide-an Efficient Reagent for Regioselective Hetero- cyclization of 3-Allyl-4hydroxy[1]benzopyran-2-ones," Synthetic Communications, Vol. 33, No. 20, 2003, pp. 36213630. doi:10.1081/SCC-120024750

[28] K. C. Majumdar and P. K. Basu, "N-Iodosuccinimide-mediated Regioselective Cyclization of $o$-cyclohex-2- enylphenols," Synthetic Communications, Vol. 32, No. 24, 2002, pp. 3719-3724. doi:10.1081/SCC-120015388

[29] K. C. Majumdar and S. Sarkaroc, " $N$-Iodosuccinimide Mediated Regioselective Heterocyclization of 3-Cyclohex-2'-enyl-4-hydroxycoumarin," Tetrahedron, Vol. 58, No. 42, 2002, pp. 8501-8504. doi:10.1016/S0040-4020(02)01024-4

[30] A. K. Yadav, B. K. Sing, N. Sing and R. P. Tripathi, “An Elegant and Unprecedented Approach to 2-methylbenzofurans," Tetrahedron Letters, Vol. 48, No. 17, 2007, pp. 6628-6632. doi:10.1016/j.tetlet.2007.07.118

[31] M. Fousteris, C. Chevrin, J. L. Bras and J. Muzart, "Water-Promoted Iodocyclisation of 2-Allylphenols," Green Chemistry, Vol. 8, No. 6, 2006, pp. 522-523. doi: $10.1039 / \mathrm{b} 602398 \mathrm{c}$

[32] V. A. Mahajan, P. D. Shinde, A. S. Gajare, M. Karthikeyan and R. D. Wakharkar, "EPZ-10 Catalyzed Regioselective Transformation of Alkenes into $\beta$-Iodo Ethers, Iodohydrins and 2-Iodomethyl-2,3-dihydro Benzofurans," Green Chemistry, Vol. 4, No. 4, 2002, pp. 325-327. doi: $10.1039 / \mathrm{b} 202725 \mathrm{a}$

[33] A. S. Gajare, M. S. Shingare and B. P. Bandgaer, "Selective Transdithio acetalization of Acetals, Ketals, Oxathioacetals and Oxathioketals Catalyzed by Envirocat EPZ10," Journal of Chemical Research (S), No. 8, 1998, pp. 452-453.

[34] K. C. Majumdar, A. Biswas and P. P. Mukhopadhyay, "Stannic Chloride-Iodine: An Efficient Reagent for Regioselective Synthesis of Furan Fused Heterocycles," Synthetic Communications, Vol. 37, No. 17, 2007, pp. 2881-2890. doi:10.1080/00397910701470982

[35] K. C. Majumdar and N. Kundu, "Effect of Substitution in Stannic Chloride-Mediated Heterocyclization of 4-Allyl3-hydroxyquinoline 2(1H) ones," Synthetic Communications, Vol. 36, No. 21, 2006, pp. 3191-3201. doi:10.1080/00397910600908892

[36] K. C. Majumdar, A. Biswas and P. P. Mukhopadhyay, "SnCl $\mathrm{S}_{4} \mathrm{I}_{2}$ Mediated Regioselective 6-Endo and 5-ExoCyclisation of Ortho-Allylenols," Canadian Journal of Chemistry, Vol. 83, No. 12, 2005, pp. 2046-2051. doi:10.1139/v05-212

[37] S. H. Mashraqui, S. Kumar and C. D. Mudaliar, "Aqueous Micellar Medium in Organic Synthesis: Alkylations and Michael Reactions of Benzotriazole," Bulletin of the Chemical Society of Japan, Vol. 74, No. 11, 2001, pp. 2133-2138. doi:10.1246/bcsj.74.2133

[38] P. V. Shinde, A. H. Kategaonkar, B. B. Shingate and M. S. Shingare, "Surfactant Catalyzed Convenient and Greener Synthesis of Tetrahydrobenzo[ $[a]$ xanthene-11-Ones at Ambient Temperature," Beilstein Journal of Organic Chemistry, Vol. 7, 2011, pp. 53-58. doi: $10.3762 /$ bjoc. 7.9

[39] F. Wang, H. Liu, L. Cun, J. Zhu, J. Deng and Y. Jiang, "Asymmetric Transfer Hydrogenation of Ketones Catalyzed by Hydrophobic Metal-Amido Complexes in Aqueous Micelles and Vesicles," The Journal of Organic Chemistry, Vol. 70, No. 23, 2005, pp. 9424-9429. doi:10.1021/jo0514826

[40] K. R. Reddy, K. C. Rajanna, S. Ramgopal, M. S. Kumar 
and S. Sana, "Environmentally Benign Synthetic Protocol for O-Alkylation of $\beta$-Naphthols and Hydroxy Pyridines in Aqueous Micellar Media," Green and Sustainable Chemistry, Vol. 2, No. 4, 2012, pp. 123-132. doi:10.4236/gsc.2012.24018

[41] P. Gogoi, P. Hazarika and D. Konwar, "Surfac$\operatorname{tant} / \mathrm{I}_{2}$ /Water: An Efficient System for Deprotection of Oximes and Imines to Carbonyls under Neutral Conditions in Water," The Journal of Organic Chemistry, Vol. 70, No. 5, 2005, pp. 1934-1936. doi:10.1021/jo0480287

[42] Y.-Y. Peng, Q.-P. Ding, Z. Li, P. G. Wang and J.-P. Chenga, "Proline Catalyzed Aldol Reactions in Aqueous Micelles: An Environmentally Friendly Reaction System," Tetrahedron Letters, Vol. 44, No. 19, 2003, pp. 3871-3875. doi:10.1016/S0040-4039(03)00692-0

[43] C. Duplais, A. Krasovskiy and B. H. Lipshutz, "Organozinc Chemistry Enabled by Micellar Catalysis. Palladium-Catalyzed Cross-Couplings between Alkyl and Aryl Bromides in Water at Room Temperature," Organometallics, Vol. 30, No. 22, 2011, pp. 6090-6097.
[44] G.-P. Lu and C. Cai, "An Odorless and Efficient Synthesis of Symmetrical Thioethers Using Organic Halides and Thiourea in Triton X10 Aqueous Micelles," Green Chemistry Letters and Reviews, Vol. 5, No. 3, 2012, pp. 481-485. doi:10.1080/17518253.2012.668221

[45] K. C. Majumdar, D. Ghosh and S. Mondal, "A Green Synthesis of Angularly Fused Furano-Pyrone/Coumarin and Quinolone Derivatives via Molecular Iodine-Mediated 5-exo-trig Cyclization in Aqueous Micelles," Synthesis, Vol. 2011, No. 4, 2011, pp. 599-602. doi:10.1055/s-0030-1258394

[46] V. K. Tandon and H. K. Maurya, "Water-Promoted Unprecedented Chemo Selective Nucleophilic Substitution Reactions of 1,4-Quinones with Oxygen Nucleophiles in Aqueous Micelles," Tetrahedron Letters, Vol. 51, No. 29, 2010, pp. 3843-3847. doi:10.1016/j.tetlet.2010.05.071

[47] M. Shiri and M. A. Zolfigol, "Surfactant-Type Catalysts in Organic Reactions," Tetrahedron, Vol. 65, No. 3, 2009, pp. 587-598. doi:10.1016/j.tet.2008.09.085 